Rev. Elev. Méd. Vét., Poys trop., 1967, 20, 3 (437-449).

\title{
Étude de la protidémie et des constantes hématologiques des camélidés en fonction des helminthes dont ils sont porteurs
}

\author{
por R. QUEVAL, M. GRABER ef Mme BRUNET
}

\begin{abstract}
RÉsuMÉ
Les auteurs étudient les variations de la protidémie et des constantes hématologiques des camélidés du Nord-Tchad, chez des animaux souffrant d'Helminthiases intestinales chroniques, à base de Nématodes principalement, et soumis d̀ un traitement au Thiabendazole.

Les résultats obtenus à plusieurs époques de l'année, avant et après traitement à des posologıes diverses, sont consignés sous forme de tableaux et de diagrammes.

Cette étude montre que les normes physiologiques ne pourront être établies valablement que lorsqu'il sera possible d'utiliser comme sujets d'étude des animaux non parasites.
\end{abstract}

\section{INTRODUCTION}

Les helminthiases du chameau au Tchad portent un lourd préjudice au potentiel économique de l'élevage camelin. Une étude récente (GRABER) (8) montre que cet animal héberge des Trématodes, des Cestodes et surtout des Nématodes appartenant aux espèces suivantes : Strongyloïdes papillosus (WEDL, 1856), Oesophagostomum columbionum (CURTICE, 1850), les Trichostrongylus vitrinus (LOOSS, 1905), Trichostrongylus probolurus (RAILLIET, 1896), Nemaiodirus spathiger (RAILLIET, 1896), Impoloia nudicollis (MÖNNIG, 1931). Hoemoncus longisfipes (RAILLIET et HENRY, 1909), et Buckleyuris globulosa (VON LINSTOW, 1901).

Par ailleurs, des investigations ont été entreprises dans le but d'opprécier la valeur anthel- minthique du Thiabendazole sur les principaux Nématodes du tractus digestıf du chameau (GRABER) (7).

Parallèlement à ces essais effectués à différentes doses et diverses époques de l'année, furent étudiées :

- les variations de protidémı avant, pendant, et après traitement,

- les modifications quantitatives du protéincgramme,

- les constantes hématologiques.

Les résultats font l'objet de cette note.

\section{RAPPEL DES TRAVAUX ANTÉRIEURS}

Peu d'études physiopathologiques ont été réalisées sur les protéines sériques des camé- 
lidés en dehors des travaux entrepris à l'Institut Pasteur de Tunis par M. KCHOUK et M. DURAND (3).

Leurs recherches ont porté au total sur 53 chameaux originaires de différentes régions de la Tunisıe.

L'analyse statıstıque des résultats (moyenne, écart type) montre que la valeur moyenne des protéines totales est de 83,6 p. $1000 \pm 2,8$, et que leur taux ne présente que de légères varıatıons suivant les zones considérées.

En même temps, il a été procédé au dosage global des protéines par la méthode colorimétrique ef au fractionnement électrophorétique. La corrélation des résultats obtenus selon ces méthodes a été appréciée.

Sur trente sérums de dromadaires mâles et femelles, les moyennes obtenues par KCHOUK ef DURAND (4) se répartissent ainsi :

- Albumine: 41,5 p. $100 \pm$. 4 p. 100.

$\alpha_{1}$ Globulines : 4,8 p. $100 \pm 1,2$ p. 100.

$\alpha_{2}$ globulines : 9,3 p. $100 \pm 2$ p. 100.

B globulines: 17,2 p. $100 \pm 1,5$ p. 100.

$\gamma$ globulines : 27,3 p. $100=3$ p. 100.

Plus récemment, DURAND et KCHOUK (1959) (5) puIs BANERJEE et Coll. (1962) (1) ont apporté leur contribution à l'hématologie du dromadaire.

Les données ainsi recuellies sont résumées dans les tableaux suivants :

a) Numérations globulaires:

1. - Globules rouges: Tableau no 1.

2. - Globules blancs: Tableau no 2 .

TABLEAU $N^{\circ} 1$

Globules rouges

\begin{tabular}{|c|c|c|}
\hline$\Delta u t e u r s$ & A n n ées & $\begin{array}{l}\text { Nombre } d^{\text {'hëmaties }} \\
\text { par } \operatorname{man}^{3}\left(x 10^{6}\right)\end{array}$ \\
\hline Croveri & 1917 & 9,0 \\
\hline Simonetta & 1917 & 12,0 \\
\hline Maliser & 1927 & 9,3 \\
\hline Ponder et Coll. & 1928 & 10,8 \\
\hline Loo Chi-Teh & 1929 & 11,8 \\
\hline Kusher & 1938 & 10,4 \\
\hline Durand et Kchouk & 1959 & 8,5 \\
\hline Banerjee et Coll. & 1962 & 7,2 \\
\hline \multirow{2}{*}{\multicolumn{2}{|c|}{$\begin{array}{l}\text { Moycnne arithmétıque } \\
\text { Erreur standard }\end{array}$}} & 9,87 \\
\hline & & $\pm 0,05$ \\
\hline
\end{tabular}

TABLEAU $N^{0} I I$

Globules blancs

\begin{tabular}{|l|c|c|}
\hline \multicolumn{1}{|c|}{ A u te urs } & An é e s & $\begin{array}{c}\text { Nombre de leucocytes } \\
\text { par } \operatorname{man}^{3}\left(\times 10^{3}\right)\end{array}$ \\
\hline Maliser & 1927 & 10,1 \\
Ponder et Col1. & 1928 & 12,0 \\
Durand et Kchouk & 1959 & 15,2 \\
Banarjee et Coll. & 1962 & 18,0 \\
\hline & & 13,8 \\
\hline
\end{tabular}


b) Formule leucocytaire: Tableau no 3.

La moyenne arithmétıque et l'écart type des résultats des travaux antérieurs ont été calculés pour connaître la valeur réelle des résultats ef les limites en dedans desquelles se maintiennent les valeurs examinées pour la majorité des sujets.

TABLEAU $\mathrm{N}^{\circ}$ III

Formule leucocytaice

\begin{tabular}{|c|c|c|c|c|c|}
\hline \multirow[b]{2}{*}{$A u t \in u r s$} & \multicolumn{3}{|c|}{ Polynucleaires } & \multirow[b]{2}{*}{ Lymphocytes } & \multirow[b]{2}{*}{ Monocytes } \\
\hline & Neutrophiles & Eosinophiles & Basophiles & & \\
\hline Yakinoff et Rastegaieff & 70,2 & - & - & 28,6 & 1,2 \\
\hline Pandez & 55,0 & 27 & 3 & 15,0 & - \\
\hline Galouzo (1929) & 52,8 & 3,0 & 1 & 42,1 & 1,1 \\
\hline $\begin{array}{c}\text { Sergent et Porcet } \\
\text { (1942) }\end{array}$ & 54,5 & 3,7 & - & 41,8 & - \\
\hline $\begin{array}{l}\text { Durand et Kchouk } \\
\text { (1959) }\end{array}$ & 58,8 & 8,1 & - & 32,2 & - \\
\hline $\begin{aligned} \text { Banerjee et Call. } & \\
& (1962)\end{aligned}$ & 51 & 6 & 0,05 & 40 & 3 \\
\hline $\begin{array}{l}\text { Moyenne arithmétique } \\
\text { et Erreur standard }\end{array}$ & $57 \pm 7$ & $5 \pm 2$ & - & $37 \pm 6$ & $1 \pm 0,5$ \\
\hline
\end{tabular}

\section{MATÉRIEL ET MÉTHODE}

\section{1) Les animaux.}

Le dromadaire ou chameau a une bosse, Camelus dromedarius, de la famille des camélidés ou Tylopodes, du sous ordre des Artiodactyles et du genre Camelus forme une importante population dans le Nord tchadien estimée à 350.000 têłes.

L'élevage du dromadaire est l'apanage des tribus nomades des régions désertiques. Lo zone dans laquelle il prospère ne dépasse guère le 14e parallèle. Au Sud de cette ligne l'humidité ef la présence d'insectes piqueurs empêchent son élevage.

\section{a) Effectif et origine.}

Au total ont été examinés 28 chameaux.

- 19 animaux originaires du Batha,

- 9 animaux originaires du Kanem.

Il faut signaler dès à présent que le parasitisme - surtout intestinal - principalement à base de Nématodes est bien plus massif au Batha qu'au Kanem.

b) Age et poids :

Les animaux utilısés sont des adultes, dans un état d'entretien médiocre et d'un poids moyen de $350 \mathrm{~kg}$.

\section{c) Etat parositare :}

Des examens coproscopiques et des cultures d'cufs ont permis d'évaluer l'importance ef la nature de l'infestation parasitaire naturelle des animaux, tout au moins en matıère de Nématodes (GRABER) (7).

\section{2) Prélèvements :}

Pour de nombreuses raisons, les prélèvements n'ont pu être effectués sur des animaux à jeun ; d'autre part, le jeûne d'un herbivore n'étant pas sans modifier ses échanges cellulaires et par conséquent, sans faire varier le taux de certains éléments de son sang, on a utılısé des animaux nourris normalement.

a) Technique de prélèvement:

Les prélèvements de sang pour les examens chimiques et hématologiques ont été falts par la voie intraveıneuse à la jugulaire. Les sérums récoltés par décantation et centrifugation sont conservés congelés.

Le prélèvement par vole endoveineuse en vue de l'examen microscopique a semblé le plus favorable pour permettre l'appréciation du 
sang, tel qu'il se trouve dans les conditions vitales.

A partir du sang ainsi recueilli, furent établis les taux de globules rouges, de leucocytes et les hémogrammes.

b) Epoques:

- Animaux originaires du Batha : juin et décembre.

- Animaux originaires du Kanem : février et novembre.

\section{3) Méthodes.}

a) Protidémie :

La détermination des protéines sériques a été réalisée de la façon suivante :

- dosage des protéines totales.

La méthode de dosage des protéines employée est celle de GORNALL, BARDAWILL et DAVID, préconisée par FLEURY (6).

Cette méthode au biuret nécessite le calcul d'un coefficient de correction, car, à côté des protéines le sérum contient toujours, en quantité varıable, d'autres substances bıurétogènes, qui apportent dans les mesures des erreurs par excès.

- Dosage des globulines.

Par précipitation avec le sulfate d'ammonium à saturation et dosage photométrique.

- Dosage des sérums albumines.

Le poids des sérums albuminés se calcule en faisant la différence entre le polds des protéines totales et celui des sérums globulines.

b) Analyse du protéinogramme :

L'appareıl à électrophorèse sur papier « JOUAN » type CNRS a 'été utilısé. Les conditıons expérimentales sont les suivantes:

- papier Arches 304.

- tampon de DURRUM de $\mathrm{pH} \mathrm{8,6,} \mathrm{de} \mathrm{force}$ ionique $0,05 \mathrm{M}$,

- durée de l'électrophorèse : 210 minutes sous une tension stabilisée de 400 volts,

- coloration au bleu de bromophénol,

- enregistrement photométrique semi-automatıque,

- décomposition de l'électrophorégramme en courbes de Gauss, de Morin.

\section{c) Techniques hématologiques:}

Les techniques sont celles qui sont admises à l'heure actuelle et employées couramment dans tous les laboratoires d'hématologie. On s'est efforcé d'obtenir, grâce aux techniques les plus simples, des résultats fidèles faciles à reproduire.

- Numérations globulaires.

La dilution du sang pour la numération des globules rouges a été faite au $1 / 200$ e dans le liquide de Marcano; celle des leucocytes au $1 / 20$ dans le liquide de dilution de Hayem.

Les numérations des hématies et des globules biancs ont été effectuées à l'aide de la cellule hématimétrique de Malassez. coloré.

- Examen microscopique du sang fixé et

L'examen microscopique de l'étalement de sang est fait après coloration selon la méthode panoptıque de MAY-GRUNWALD-GIEMSA.

\section{RÉSULTATS}

\section{1) Protidémie.}

L'étude a été réalisée à partir de chameaux plus ou moins parasıtés (GRABER, 1966) (7) et soumis à une médication anthelminthique. Les dosages de proténes totales, d'albumine et de globulines furent effectués avant et après traitement.

Dix-neuf animaux venus du Batha furent séparés en deux lots dont I'un reçut $300 \mathrm{mg} / \mathrm{kg}$ de Thiabendazole pur en juin 1965, et le second $400 \mathrm{mg} / \mathrm{kg}$ en décembre.

Des doses de 70 et $100 \mathrm{mg} / \mathrm{kg}$ (février 1965) et $300 \mathrm{mg} / \mathrm{kg}$ (en octobre) furent distribuées à des dromadaires originaires du Kanem.

Le toux protémique du chameau normal (g p. 1.000) semble être le suivant:

$\begin{array}{ccc}\text { Protéines totales } & \text { Albumine } & \text { Globulines } \\ -\overline{83,9} & \overline{34,8} & - \\ & & 49,1\end{array}$

a) Chameaux du Kanem, février 1965 : Tableau IV. 
TABLEAU $N * I V$

Chameaux du Kanem - Füvrier 1965

Traités au Thiabendazole pur

\begin{tabular}{|c|c|c|c|c|c|}
\hline \multirow{2}{*}{$\begin{array}{c}\mathrm{N}^{\circ} \\
\text { des } \\
\text { animaux }\end{array}$} & \multirow{2}{*}{$\begin{array}{c}\text { Dose } \\
\text { administrée } \\
\text { mg } / \mathrm{kg}\end{array}$} & & \multicolumn{3}{|c|}{ Pratéines sëriques (en $\mathrm{gr}, 0 / 00$ ) } \\
\hline & & & Totales & Albumines & Globuilines \\
\hline 6 & 70 & $\begin{array}{l}\text { Avant traitement } \\
\text { Après traitement }\end{array}$ & $\begin{array}{l}63,87 \\
60,71\end{array}$ & $\begin{array}{l}18,1 \\
22,2\end{array}$ & $\begin{array}{l}45,72 \\
38,5\end{array}$ \\
\hline 7 & 70 & $\begin{array}{l}\text { Avant traitement } \\
\text { Aprẽs traitement }\end{array}$ & $\begin{array}{l}70,2 \\
70,2\end{array}$ & $\begin{array}{l}20,1 \\
20,1\end{array}$ & $\begin{array}{l}50,0 \\
50,1\end{array}$ \\
\hline 8 & 70 & $\begin{array}{l}\text { Avant traitement } \\
\text { Aptēs traitement }\end{array}$ & $\begin{array}{l}67,1 \\
70,2\end{array}$ & $\begin{array}{l}22,2 \\
22,2\end{array}$ & $\begin{array}{l}44,9 \\
48,0\end{array}$ \\
\hline Moyennes & 70 & $\begin{array}{l}\text { Avant traitement } \\
\text { Après traitement }\end{array}$ & $\begin{array}{l}67,0 \\
67,0\end{array}$ & $\begin{array}{l}20,2 \\
21,5\end{array}$ & $\begin{array}{l}46,6 \\
45,5\end{array}$ \\
\hline 1 & 100 & $\begin{array}{l}\text { Avant traitement } \\
\text { Après traitement }\end{array}$ & $\begin{array}{l}67,1 \\
73,5\end{array}$ & $\begin{array}{l}20,17 \\
20,17\end{array}$ & $\begin{array}{l}46,93 \\
53,35\end{array}$ \\
\hline 4 & 100 & $\begin{array}{l}\text { Avant traitement } \\
\text { Après traitement }\end{array}$ & $\begin{array}{l}67,1 \\
67,1\end{array}$ & $\begin{array}{l}22,2 \\
18,1\end{array}$ & $\begin{array}{l}44,9 \\
48,9\end{array}$ \\
\hline Moyennes & 100 & $\begin{array}{l}\text { Avant traitement } \\
\text { Après traitement }\end{array}$ & $\begin{array}{l}67,1 \\
70,3\end{array}$ & $\begin{array}{l}22,2 \\
19,1\end{array}$ & $\begin{array}{l}45,9 \\
51,1\end{array}$ \\
\hline
\end{tabular}

\section{COMMENTAIRES}

Les dosages des protéines après traitement à $70 \mathrm{mg} / \mathrm{kg}$ ne montrent globalement aucune variation.

En effet, à $70 \mathrm{mg} / \mathrm{kg}$ sur les animaux fortement parasités l'efficacité du Thıabendazole est nulle sur les Haemoncus longistipes ; par contre, à cette dose, lorsque les chameaux sont faiblement parasités, et les parasites sexuellement mûrs, l'efficacité est totale (100 p. 100) sur Haemoncus longıstipes et Strongyloides papillosus.

Les animaux tratés à la dose de $100 \mathrm{mg} / \mathrm{kg}$ montrent une diminution du taux d'albumine et un accroissement des globulines qui tendent

\section{TABLEAU $N^{\circ} \mathrm{V}$}

Moyenne de quatre animaux traités à 1a dose de 300 mg de Thlabendazole pur par kg

\begin{tabular}{|l|c|c|c|}
\hline \multirow{2}{*}{} & \multicolumn{3}{|c|}{ Protêines sériques (en $\mathrm{g}$ 0/00) } \\
\cline { 2 - 4 } & Totales & Albumines & Globulines \\
\hline Avant traitement & 73,8 & 26,1 & 47,7 \\
Après traitement & 85,4 & 28,6 & 56,8 \\
\hline
\end{tabular}

à un taux normal. Les protéines totales sont inférieures à la normale.

Du point de vue protıdémie, ces résultats peu orthodoxes peuvent s'expliquer par l'infestation massive par Cestodes et Trichures.

b) Chameaux du Kanem : octobre 1965, tableau $n^{\circ} \mathrm{V}$.

\section{COMMENTAIRES}

A cette période le polyparasitısme est important et particulièrement favorable au développement des Haemoncus (de 300 à 3.500) sexuellement immatures. Les fourrages assurent une ration d'entretien suffisante.

A la dose de $300 \mathrm{mg} / \mathrm{kg}$, le taux d'efficacité du Thiabendazole sur des Cestodes tels que Maniezia expansa, Avitellina centripunctata et Avitellina woodlandi est respectivement de 8,8 p. 100 dans le premier cas, ef de 45,5 p. 100 dans le second. Par ailleurs, le traitement élimine en totalité (100 p. 100) les Nématodes d̀ l'exception d'Haemoncus longistipes chez les animaux fortement parasités où le taux d'efficacité n'est que de 85 p. 100. 
Du point de vue biologique, on note une nette augmentation des protéines totales ( +15 p. 100) dont le taux devient normal. Ces résultats sont d̀ mettre en parallèle avec une augmentation du nombre des hématies $(+10$ p. 100), et un gain de poids ( 11 p. 100).

c) Animaux du Batha, juin 1965, Tableau no $\mathrm{VI}$.

TABLEAU $\mathrm{N}^{\circ} \mathrm{VI}$

Moyenne de douze anỉmaux traitês à la dose de $150 \mathrm{mg}$ de Thtabendazole pur par $\mathrm{kg}$

\begin{tabular}{|c|c|c|c|}
\hline \multirow{2}{*}{} & \multicolumn{3}{|c|}{ Protéines sériques (en g 0/00) } \\
\cline { 2 - 4 } & Totales & Albumines & Globulines \\
\hline Avant traitement & $71,75 \pm 4,6$ & $24,6 \pm 1,6$ & $47,1 \pm 4,5$ \\
Aptès traitement & 83,1 & 29,9 & 33,2 \\
\hline
\end{tabular}

\section{COMMENTAIRES}

En juin, le nombre d'œufs, au gramme, est fort élevé, l'infestation massive. Par exemple, le nombre d'Haemoncus rencontré par anımal est d'environ 1.300 , et beaucoup sont sexuellement Immatures.

D'où, avant traitement, un faible taux de protéines, accompagné d'une anémie et d'un médiocre état physiologique.

Le traitement a pour effet de détruire la totalité des Trichostrongylus et des Impalä̈a, mais non les Haemoncus, qui persistent, surtout s'ils sont immatures. Néanmorns, le Thiabendazole à $150 \mathrm{mg} / \mathrm{kg}$ dissocie l'association de Nématodes la plus dangereuse c'est-à-dire Hoemoncus + impalaĩo et Trichostrongylus. L'un des facteurs d'amaigrissement disparaît, et l'animal réagit immédiatement dans un sens favorable. Cependant la dose de $300 \mathrm{mg} / \mathrm{kg}$ doit être préconisée car du point de vue traitement, elle se montre la plus polyvalente.

d) Un dernıer essai a été réalisé en décembre 1965 à parti de quatre dromadaires $(t)$ originarres du Batha.

A cette époque le polyparasitisme est bien inférieur à ce qu'il est au début ou à la fin de la saison des pluies. Généralement, on ne rencontre que 150 Haemoncus par animal et souvent, ils sont sexuellement môrs.

Aussi avant traltement, les protéines totales sont-elles proches de la normale et après traitement, les protéınes restent pratiquement stables. Corrélativement on ne note qu'un faible accroissement pondéral $(+2$ p. 100). e) Cette série d'exemples démontre que le parasitisme gastro-intestinal entraîne des modifications sensibles des protéines du sang : le déficit en protélnes totales peut atteindre 15 p. 100. Les albumines baissent au profit des globulines. Le traitement antıparasitaire semble rétablir progressıvement l'équilibre normal.

\section{2) Analyse du protéinogramme.}

Les investıgations ont porté sur un lot de dromadaires venus du Batha et faiblement parasités ainsi qu'il a été dit plus haut (loł de décembre 1965).

Bien qu'après traitement, les protéines tatales demeurent à peu près stables, l'étude des fractions électrophorétiques révèle de sensıbles modifications quantitatives.

Après traitement, on note, en règle générale, une nette augmentation de la sérum albumıne, et un léger accroissement des $\beta$ globulines. Par contre, on observe une chute des $\alpha 2$ et $\gamma$ globulines (Tableau no $\mathrm{VII}$ ).

Comparativement aux résultats des auteurs tumisiens KCHOUK et DURAND, la fraction albuminique est encore faible et les globulınes élevées.

Ces taux pourraient être attribués à la présence de Cestodes tels que Stilésio globipunctata (25 p. 100) et Echinococcus polymorphus (35,6 p. 100).

(*) Ayant reçu $400 \mathrm{mg} / \mathrm{kg}$ de Thiabendazole. 


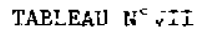

Fractions électrophorètiques des protëines sériques

\begin{tabular}{|c|c|c|c|c|c|}
\hline \multirow{2}{*}{$\begin{array}{l}\text { Protéines } \\
\text { Totales. }\end{array}$} & \multirow[t]{2}{*}{ Albumines } & \multicolumn{4}{|c|}{ G $1 \circ b$ u lines } \\
\hline & & $a 1$ & $a 2$ & $\beta$ & $\gamma$ \\
\hline $\begin{array}{c}\text { Avant traitement } \\
100 \mathrm{p} .100\end{array}$ & 26,3 & 7,7 & 12,6 & 25,9 & 37,4 \\
\hline $79,8 \mathrm{~g} \mathrm{o/00}$ & 20,9 & 6,2 & 10,1 & 12,7 & 29,9 \\
\hline $\begin{array}{l}\text { Après traitement } \\
100 \mathrm{p} .100\end{array}$ & 37,0 & 7,4 & 9,4 & 18,1 & 28,0 \\
\hline 80,5 g $0 / 00$ & 29,8 & 6,0 & 7,6 & 14,5 & 22,6 \\
\hline $\begin{array}{l}\text { Référence } \\
100 \mathrm{p} \cdot 100\end{array}$ & 41,5 & 4,8 & 9,3 & 17,2 & 27,3 \\
\hline 33,9 g $0 / 00$ & 34,8 & 4,0 & 7,8 & 14,5 & 22,6 \\
\hline
\end{tabular}

\section{3) Résultats hématologiques.}

a) Numérations globulares:

Le nombre de globules rouges et blancs contenus dans le sang par millimètre cube est variable suivant les races, le sexe, l'alimentation, l'état de sonté, et le climat. Ces facteurs expliquent que les chiffres donnés par divers auteurs soient fort différents les uns des autres.

La particularité du sang de chameau est la forme générale des globules rouges qui sont ovalaires ou elliptiques comme ceux des anımaux à sang froid. Mais à l'inverse de ce que I'on observe chez ces derniers, il n'y a pas de noyau.

Les dimensions moyennes sont de $8 \mu$ sur $4 \mu$ et $2 \mu$ d'épaisseur.

Les valeurs moyennes des numérations globulaires sont réunies dans le tableau suivant : no VIII.

TABLEAU $\mathrm{N}^{\circ} \mathrm{VIII}$

Numérations globulaires avant et après traitement au Thiabendazole pur

\begin{tabular}{|c|c|c|c|c|c|}
\hline $\begin{array}{l}\text { Origine } \\
\text { des } \\
\text { arimaux }\end{array}$ & $\begin{array}{l}\begin{array}{c}\text { Epoque } \\
\text { des } \\
\text { interventions }\end{array} \\
\text { incen }\end{array}$ & $\begin{array}{c}\text { Dose } \\
\text { administrée } \\
(\text { en mg } / \mathrm{kg} \text { ) }\end{array}$ & & Hénaties & Leucacytes \\
\hline Капеm & Fêvrier & 70 et 100 & $\begin{array}{l}\text { Avant traitement } \\
\text { Après traitement }\end{array}$ & $\begin{array}{r}10.464 .000 \\
9.000 .000\end{array}$ & $\begin{array}{r}7.720 \\
18.380\end{array}$ \\
\hline Kanem & Octobre & 300 & $\begin{array}{l}\text { Avant traitement } \\
\text { Aprẽs traitement }\end{array}$ & $\begin{array}{l}8.000 .000 \\
8.900 .000\end{array}$ & $\begin{array}{l}\text { I-1.3 } 3 \mathrm{CO} \\
12.600\end{array}$ \\
\hline Batha & Décembre & 400 & $\begin{array}{l}\text { Avant traitement } \\
\text { Après traítement }\end{array}$ & $\begin{array}{r}9.360 .000 \\
10.725 .000\end{array}$ & $\begin{array}{l}27.650 \\
37.750\end{array}$ \\
\hline
\end{tabular}

\section{COMMENTAIRES}

De la lecture de ce tableau, il ressort que pour les anımaux peu anémiés et traités à faibles doses (70 ef $100 \mathrm{mg} / \mathrm{kg}$ ), it y $a_{1}$ après traitement, une chute du nombre des hématies et une augmentation des leucocytes.

Sur les animaux anémıés, fortement parasités et traités à fortes doses (300 et $400 \mathrm{mg} / \mathrm{kg}$ ) le nombre de globules rouges augmente nettement.

b) Formule leucocytare:

S'il est un fait que la formule leucocytaire ou hémogramme est très variable suivant les individus, elle est également variable avec le moment de la journée, la technıque et l'endroit de la prise de sang. 
Ces faits suffisent à expliquer la divergence des résultats principalement en ce qui concerne granulocytes et monocytes.

Cependant, il est possible d'obtenir une bonne moyenne de chaque type de leucocytes en multiplıant les observations et en les réalisant toutes dans des conditions identiques.

Enfin, malgré les variations normales individuelles ou journalières observées chez un même individu, on constate au cours de parasitoses ou d'infections pathologiques diverses des variations caractéristiques, nettes ef constantes de l'hémogramme.

La morphologie des leucocytes du chameau ne présente pas une grande originalité et de ce fait ne nécessite nullement une description détaillée.

Les polynuclécires neutrophiles qui interviennent dans tous les phénomènes de phagacytose mesurent environ $13 \mu$ de diamètre et ont une forme constante.

Les granulocytes éosinophiles ont un diamètre de $11 \mu$ environ et leur forme générale irrégulière.

Les polynucléaires basophiles mesurent environ $10 \mu$ de diamètre

Parmi la série agranulocytaire, les lymphocytes mesurent environ $8 \mu$ de diamètre, le contour est net, le noyau arrondi de situation légèrement excentrıque remplit presque toute la cellule.

Les monocytes ont un diamètre de $13 \mu$ environ.

On rencontre également des cellules mononuclées auxquelles la basophilie accentuée de leur cytoplasme, l'aspect clair ef morgansé de leur noyau, la présence inconstante de nucléoles, confèrent l'allure générale des formes jeunes, mais leur nature lymphocytaire, mononucléaire ou monocytaire est impossible à préciser.

Les résultats observés ont été consignés sur des diagrammes où chaque sujet est figuré par un point. En abscisse est portée l'origine des animaux, et en ordonnée, le pourcentage de cellules observées.

Les diagrommes permettent les observations suivantes:

- Les animaux originaires du Batha ef du Kanem, présentent au mols de juin et d'octobre les pourcentages les plus élevés de polynucléaires neutrophiles $(66,4$ et 51,0 p. 100).

- Les prélèvements réalisés au Batha en décembre et au Kanem en février montrent un pourcentage identique de polynucléaires neutrophiles ( 45,5 et 44,6 p. 100 ).

II est facile de voir que l'éosinophilie est surtout marquée chez les animaux originaires du Kanem $(14,4$ et 10,9 p. 100), contre (4,9 et 2,8 p. 100) pour les animaux vivants au Batha. Cette éosinophilie est le reflet des pourcentages. moyens d'infestation parasitaire: 31,3 p. 100 au Kanem contre 23,0 p. 100 au Batha.

- En février et décembre, aussi bien au Kanem qu'au Batha lorsque le polyparasitisme est bien inférieur à ce qu'il est au début (juin) ou en fin (octobre) de saison des pluies, on note un taux supérieur de lymphocytes dans le sang circulant $(44,9$ et 39,5 p. 100) contre $(33,7$ et 32,0 p. $100)$ en octobre et juin, périodes de fortes préclpitations, tant au Batha qu'au Kanem.

Le tableau n० $1 X$ résume les valeurs moyennes enregistrées avant et après traitement au Thiabendazole.

TABLEAU IX. - Formule leucocytaire - Valeur moyenne avant et aprés traitement au Thiabendazole pur

\begin{tabular}{|c|c|c|c|c|c|c|c|}
\hline $\begin{array}{l}\text { Origine } \\
\text { des } \\
\text { animaux }\end{array}$ & $\begin{array}{c}\text { Epoque } \\
\text { des } \\
\text { interventions }\end{array}$ & $\begin{array}{l}\text { Dose } \\
\text { administrée } \\
\text { mg } / \mathrm{kg}\end{array}$ & & Neutrophiles & Eosinophiles & Lymphocytes & Monocytes \\
\hline Kanem & Fêvrier & 70 et 100 & $\begin{array}{l}\text { Avant } \\
\text { Après }\end{array}$ & $\begin{array}{l}44 \\
45\end{array}$ & $\begin{array}{l}10,5 \\
10\end{array}$ & $\begin{array}{l}44,5 \\
42\end{array}$ & $\begin{array}{l}1 \\
3\end{array}$ \\
\hline Kanem & Octobre & 300 & $\begin{array}{l}\text { Avant } \\
\text { Après }\end{array}$ & $\begin{array}{l}54 \\
47\end{array}$ & $\begin{array}{r}19 \\
9\end{array}$ & $\begin{array}{l}27 \\
44\end{array}$ & - \\
\hline Batha & Décembre & 400 & $\begin{array}{l}\text { Avant } \\
\text { Aprẽs }\end{array}$ & $\begin{array}{l}54 \\
60\end{array}$ & $\begin{array}{l}9 \\
2\end{array}$ & $\begin{array}{l}37 \\
38\end{array}$ & - \\
\hline Yoyennes & & & $\begin{array}{l}\text { Avant } \\
\text { Aptès } \\
\text { Rếf. }\end{array}$ & $\begin{array}{c}52 \\
50,7 \\
57^{ \pm} 7\end{array}$ & $\frac{12}{7} 5^{2} \pm 2$ & $\begin{array}{l}36 \\
41,3 \\
37 \pm 6\end{array}$ & $\overline{1}_{1 \pm 0,5}^{-}$ \\
\hline
\end{tabular}




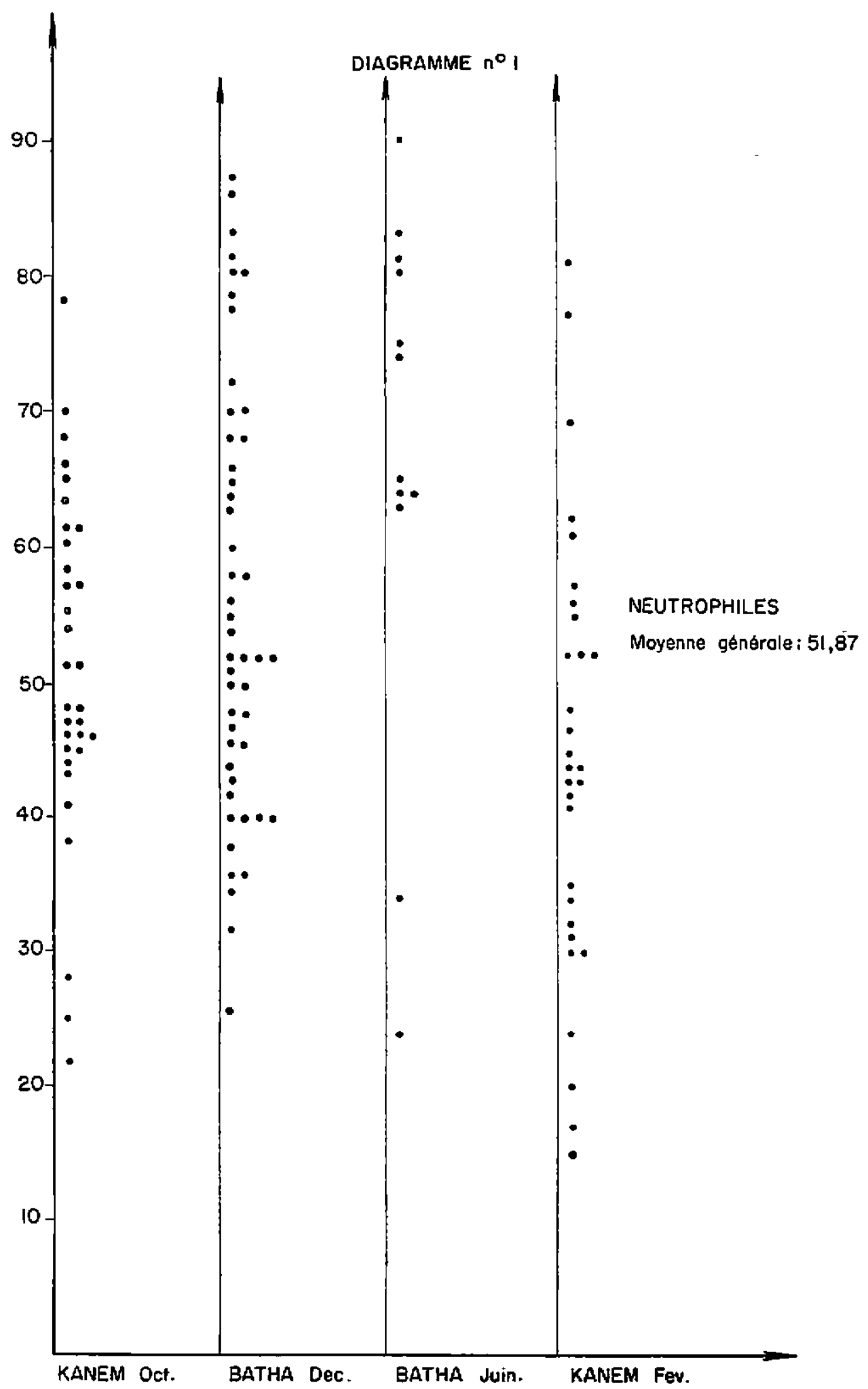




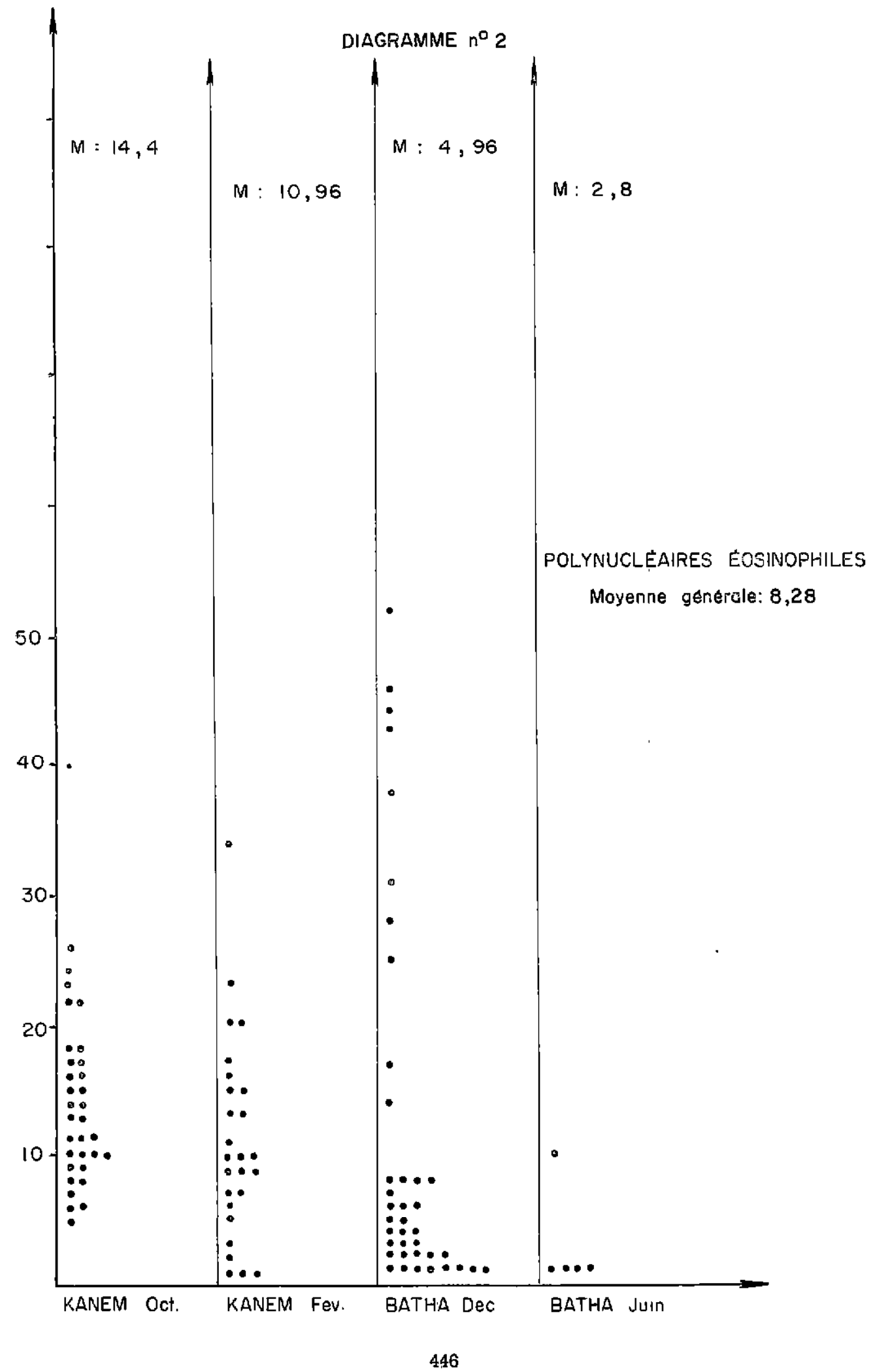




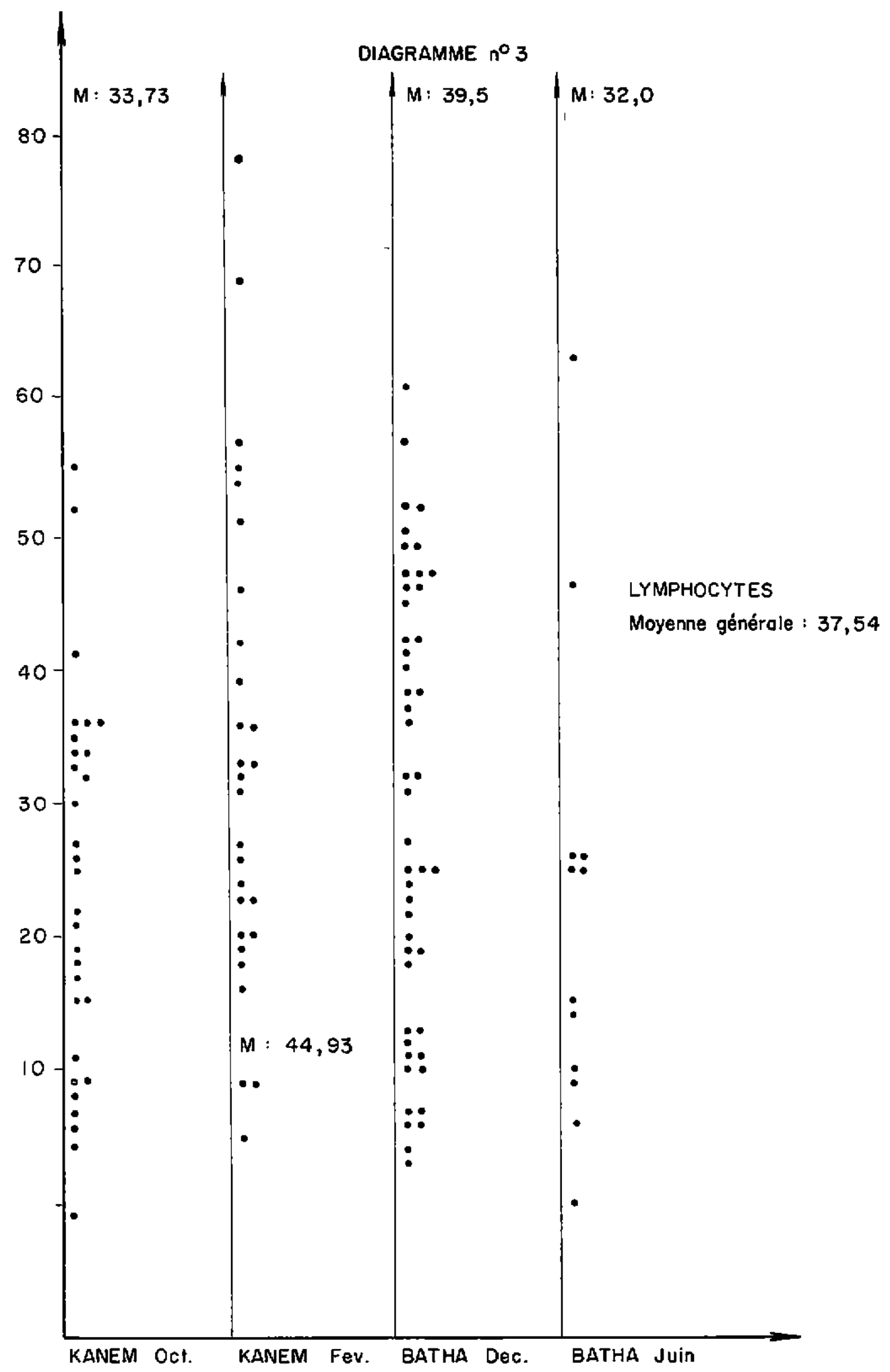


Les résultats obtenus sur les animaux du Kanem aux doses de 70 et $100 \mathrm{mg} / \mathrm{kg}$ sont négatıfs, surtout en raison des conditions alimentaires défavorables.

Aussi, les constantes hématologiques sont-elles Identiques, en particulier l'éosinophilie.

En octobre, où l'infestation parasitaire est massive ef l'alimentation satisfasante, le traitement anthelminthique à la dose de $300 \mathrm{mg} / \mathrm{kg}$ provoque une chute de l'éosinophilie de l'ordre de 47 p. 100, ef une augmentation des lymphocytes.

Au Batha, où le parasitisme est, à partir de décembre relativement faıble, le traitement à la dose de $400 \mathrm{mg} / \mathrm{kg}$ ramène à la normale le nombre des polynucléaires éosinophiles.

Sur des animaux fortement parasités (GRABER) (8), les essais de traitement au Thiabendazole montrent donc à quel point «les standards hématologiques» peuvent s'éloıgner de ce qu'il est convenu d'appeler la «normale».

\section{CONCLUSION}

Les chameaux que I'on rencontre en République du Tchad, d'aires géographiques différentes et de races diverses, présentent à certaines époques de l'année une nette hypoprotidémie ainsi qu'à une anémie globulaire.

Les recherches faltes parallèlement révèlent la présence dans le tractus digestif de nombreux helminthes. Les plus fréquents sont des Cestodes et surtout des Nématodes (Haemoncus longistipes, Trichostrongylus sp. ef Impalaia nudicollis).

Sous l'influence d'une nématodicide tel que le Thiabendazole, à la posologie de 300 à $400 \mathrm{mg} /$ $\mathrm{kg}$, on observe déjà la régression de l'anémie et une nette augmentation des protéines totales. Malheureusement, le traitement n'est pas polyvalent.

II en résulte que les constantes brochimiques et hématologiques ne peuvent dans les circonstances actuelles être établies sưrement. Elles le seront quand il sera possible d'obtenir des animaux non parasités.

\section{SUMMARY}

Study of the protidemia and the blood characteristics of camels according to the various species of parasitic helminths

The variations of the protidemia and the blood characteristics of camel from Northern Chad have been studied in animals, chronically infested by intestinal helminths, mainly nematods, and given a treatment with Thiabendazole.

The resulis recorded in several times of the year, before and after the treatment at various doses are presented in tables and diagrams.

This study shows that the physiological characteristics can only be established when parasited-free animais are used for the experiments.

\section{RESUMEN}

Estudio de la protidemı y de las constantes hematologicas de los camellos en función de los helmíntos que les parasitan

Los autores estudian las modificaciones de la protidemia y de las constantes hematologicas de los camellos en el norte de Chad, en animales atacados por helmintiasis intestinales crónicas, principalmente con nemátados, y tratados con thiabendazolo.

Se notan los resultados, bajo forma de cuadros y diagramas, obtenidos en varios momentos del año, antes y despues del tratamiento en diversas posologias.

Este estudio demuestra que se podra determinar válidamente las normas fisiologicas solo cuando sera posible utilizar animales sın parásitos como anima! testigo. 


\section{BIBLIOGRAPHIE}

1. BANERJEE (S.), BHATTACHARJEE (C.), et SINGH (T. I.). - Hematological studies in the normal adult Indian camel (comelus dromadorius).

2. CURASSON. - Le chameau ef ses maladies. Paris Vigot frères, 1947.

3. KCHOUK (M.) et DURAND (M.). - Quelques dosages chimiqués dans le sang des dromadaires en Tunisie. Arch. Inst. Pasteur Tunis, 1958, 35 (1), 3.

4. DURAND (M.) et KCHOUK (M.). - Le « krafft » une ostéopathie dystrophique du dromadaire. Arch. Inst. Pasteur Tunis, 1958, $35(2), 107$.

5. DURAND $\left(M_{\text {. }}\right.$ ) et KCHOUK (M.). - Quelques constantes hématologiques chez le dromadaire tunisien. Arch. Inst. Posteur Tunis, 1959, 36 (2), 183.
6. FLEURY (P.). - Fiches techniques de chimie biologique. 5e supplément. Paris ( 175. boulevard Saint-Germain), Vega, 1953.

7. GRABER (M.). - Etude dans certaines conditions africaines de l'action antiparasitaire du Thiabendazole sur divers Helminthes des animaux domestiques II dromadaire. Rev. Elev. Méd. vét. Pays trop. 1966. 4 (sous presse).

8. GRABER (M.). - Enquêtes sur les Helminthes du dromadaire tchadien. Efude des «strongyloses 》 gastro-intestinales et de l'Haemoncose à Haemoneus longistipes. Rev. Elev. Méd. Vét. Pays Trop. 1967, à paraître

9. RECEVEUR (P.). - Rapport Annuel du Service de l'Elevage., 1944. 